\title{
Simplified teixobactin analogues to target superbugs
}

\author{
Ishwar Singh*,1 \\ ${ }^{1}$ School of Pharmacy, JBL Building, University of Lincoln, Green Lane, Lincoln, LN6 7DL, UK \\ * Author for correspondence: Tel.: +44 1522 886915; Isingh@lincoln.ac.uk
}

Ishwar Singh speaks to Benjamin Walden, Commissioning Editor. Ishwar Singh is Senior Lecturer in biological chemistry at the School of Pharmacy, University of Lincoln. Prior to Lincoln, he had held many prestigious fellowships such as the Alexander von Humboldt fellowship, Germany; and Senior Research Fellowship, DANIDA, Denmark and CSIR, India. He is an organic chemist. He has developed bioconjugations for DNA, RNA and polymer modifications. He is currently leading research in novel antimicrobials based on rational design against clinically important resistant bacteria (such as methicillin-resistant Staphylococcus aureus, vancomycin-resistant enterococci, mycobacteria, Klebsiella pneumoniae, Acinetobacter baumannii and Pseudomonas aeruginosa), biologics delivery, peptides, sequence-selective DNA cross linking, nanoparticles modifications for drug delivery and diagnostic applications.

First draft submitted: 19 October 2017; Accepted for publication: 19 October 2017; Published online: 15 December 2017

Keywords: MRSA • resistant bacteria $\bullet$ teixobactin $\bullet$ teixobactin analogues

Tell us a little bit about yourself and your career to-date?

I am a Senior Lecturer in biological chemistry at the School of Pharmacy, University of Lincoln. I am an Organic Chemist by training. Prior to Lincoln, I had held many prestigious fellowships such as the Alexander von Humboldt fellowship, Germany; and Senior Research Fellowship, DANIDA, Denmark and CSIR, India.

Tell us about the current research projects that are ongoing in your lab?

The key focus of our research is to use chemistry as a tool to understand fundamental biological questions. We use such understanding to provide potential solutions to current challenges. We make designer molecules. We use these designer molecules for diverse applications such as antibiotics, drug delivery, DNA diagnostics, and nanotechnology. For antibiotics, we are mainly focusing on resistant bacteria.

Talking specifically about the teixobactin project, what was the rationale behind this research that led to the paper?

Teixobactin producing bacteria did not make teixobactin for human use. The rationale behind the paper is very simple. There is a need for new potent antibiotics. We are dissecting the teixobactin to understand how it works, and using this knowledge to make simpler and potent analogues. Such analogues are critical to realise the therapeutic potential of teixobactins for human use. There is a high failure rate in translating a molecule from discovery stage to clinic due to common drug development challenges such off target toxicity in humans. Our focus has been to address these challenges by providing a library of simpler but potent analogues against superbugs such as MRSA. We have previously developed the highly efficient synthesis and first structure-activity relationship understanding of new game-changing antibiotic teixobactin. We have defined the first three-dimensional structures, the roles on amino acids and their impact on biological activity. In the paper, we have focused on the key bottleneck of teixobactin development, enduracididine. We have replaced challenging enduracididine with its isoestres, which are commercially available and easier to make.

Can you tell us the key findings of this project?

We have used commercially available building blocks, rather than the synthetically challenging building block enduracididine, to make eight analogues of teixobactin. All showed potent activity against MRSA. Importantly, teixobactin works by unique modes of action which are not susceptible to current resistance mechanisms. 


\section{Can you explain the key steps in your synthesis of the teixobactin analogues?}

In natural teixobactin, a key amino acid, enduracididine, is very difficult to synthesize. We propose instead modifying commercial available amino acids, such as lysine and arginine. By converting from an amino to guanidine functionality in a single step, we managed to diversify the analogues with similar structure to enduracididine.

\section{Can you tell us about the predicted mechanism of action of this drug?}

The mechanism of action of teixobactin is still an open project. The current understanding is that it operates by more than one mechanisms of action. It binds to highly conserved targets (pyrophosphate motif of lipid II and lipid III). These targets are essential for bacterial survival and not known for mutation, a major reason to resistance. Vancomycin, a clinically used antibiotic, also binds to lipid II using peptide motif which is known for mutations. It took nearly 30 years of use to see any resistance against vancomycin.

\section{What can do you prevent resistance from developing against this drug? Has it been tested in} combination therapy?

Rigorous resistance screening against diverse pathogenic bacteria. A library of teixobactin analogues and good understanding of how teixobactin works could be very useful to address the challenge from potential resistance. It is most likely to last longer than vancomycin because of its unique modes of action. It has not been tested in combination therapy yet.

\section{Apart from drug resistance, what do you find the greatest challenge in this field at the moment? (And how do you think we need to go about addressing these issues?)}

Lack of molecules which work by new modes of action is a great challenge and this is one reason behind a lot of excitement around teixobactin. We need to develop libraries of lead molecules and discover more molecules with new modes of action. There is a need for collaborations with different disciplines as well as academia and industry and public-sector institutes to work together to bring new antibiotics.

As with all drug discoveries, a key challenge is the translation of success from the discovery phase to therapeutic use of teixobactins in humans. Despite recent calls for action from the WHO, AMR review recommendations (UK Government), antibiotic development research is still underfunded.

Are there any specific technological advances that have revolutionized your work recently, or will revolutionize it in the future?

The development of high throughput platform to identify new antibiotics scaffolds would also be great.

Looking to the future, where do you imagine your work going in the next 5-10 years?

In this timescale, I would to love to see to see our molecules advancing towards the clinic.

Finally, if you had unlimited money and resources, what would you make it your priority to do in this field?

It's an unrealistic question, because if there is unlimited money available, there is no need for any priority. With limited funding, I would like to support more small-scale projects to get the proof of concept tested. In second stage to develop consortia/s on the basis of proof of concepts rather than funding large-scale projects directly. If possible for small-scale projects, I would like to anonymize the applicants in initial screening to support the brightest ideas. This process potentially will enable the development of small projects and their applications to address real-world challenges.

\section{Financial \& competing interests disclosure}

The author has no relevant affiliations or financial involvement with any organization or entity with a financial interest in or financial conflict with the subject matter or materials discussed in the manuscript. This includes employment, consultancies, honoraria, stock ownership or options, expert testimony, grants or patents received or pending, or royalties.

No writing assistance was utilized in the production of this manuscript.

\section{Disclaimer}

The opinions expressed in this interview are those of the interviewee and do not necessarily reflect the views of Future Science Ltd. 